\title{
THE PLACE OF RESPIRATORY FUNCTION TESTS IN CLINICAL MEDICINE
}

\author{
D. V. Bates, M.D., M.R.C.P. \\ First Assistant, Medical Unit, St. Bartholomeac's Hospital, London, E.C.I
}

\section{Introduction}

It is difficult to settle on a precise date for the introduction of objective methods of measurement into clinical medicine. Perhaps a good case can be made out for the year 1867 when Clifford Allbutt arranged for the first clinical thermometer to be manufactured. This technique survived the ridicule with which it was greeted, but perhaps even then it was realized that the substitution of a thermometer for the sensitive hand of the physician was a dangerous precedent, likely eventually to lead to the substitution of one technique of diagnosis by another. It is important that certain erroneous preconceptions are cleared away therefore, before the particular question of the place of respiratory function tests is considered. The days when a vain search was made for tests which were infallible in the diagnosis of this or that condition, have passed. It is quite clear that the best contemporary practice assembles its information, the history, the physical examination, the $\mathrm{X}$-ray, the blood count, etc., with care and with knowledge of what may be misleading in each of them; and then reaches a diagnosis by putting this information together as neatly and as coherently as it will allow, guided by its experience. No-one expects the blood urea to diagnose chronic nephritis, so there need be no surprise if the vital capacity does not diagnose emphysema. Further one can recall that all investigations, from the measurement of the body temperature onwards, have started essentially as research investigations used only by one or two laboratories by one or two physicians. A general application of the method has followed a general understanding of its usefulness. Some contemporary investigations are in the border country between being ' research' and 'clinical' investigations;' the electroencephalogram, the serum lipo-proteins perhaps being examples of these. To the question, there fore, should every hospital have facilities for studying the serum lipo-proteins, the answer might be that it should be available in any department where vascular disease is particularly studied, but not necessarily elsewhere. The seems little doubt that the full range of cogtemporary respiratory function tests should $\mathrm{B}$. available to every chest hospital, but this is not the same as suggesting that every hospital should hade a pulmonary function laboratory.

Lastly, there are some difficulties that partio cularly apply to respiratory function tests. The co-operation of the patient is required, though this very rarely presents a problem. Indeed $-I$ have come to the conclusion that if the patiegt complains of dyspnoea, the idea of measuring hos lung performance seems at the moment robes logical and reasonable to the patient than it do the physician. Some of the recent techniques of measurement of respiratory function are comp cated and all of them require trained staff. "We need not be deterred by the fact that these tests cannot be applied in every doctor's consultiong room. The value of simple tests, like the maxima breathing capacity, tends to be overstresse्fid because they can be easily measured, and it most important that the practical value of the te्डst in clinical practice receives very critical attentiogi, since if nothing is going to be learned of real val to the physician, any test, however simple, is waste of time.

The last ten years have seen the completion ofa great deal of the essential foundation work in the study of pulmonary physiology. These advances have been most excellently brought together and simply described in a recent book by J. H. Comr参 and his colleagues. The purpose of this artiote is, firstly, to describe contemporary tests of function and to discuss the information they aq capable of yielding; and secondly to discuss the clinical situations in which this information $Y_{s}$ important.

\section{What Aspects of Pulmonary Function can bo Measured?}

Trotter in 1933, pointed out that 'up to the present time, medicine has almost wholly avoid the burden of measurement.' Recent researç 
has given us an exact knowledge of what constitutes normal lung function, and what the derangements are in certain diseases. The following ' compartments' of pulmonary function can be separately measured.

I. Total ventilation-ability to move air normally in and out.

2. Measurement of the subdivision of lung volume-volume of gas normally 'residual' or displaceable.

3. Measurement of evenness of gas distribution within the lungs - the air breathed in is almost perfecly divided between alveoli in normal lungs.

4. Measurement of physical characteristics of the lungs-normally the lungs are easily distensible, and the bronchial resistance to airflow is low.

5. Measurement of the efficiency of gas transfer across the lung-measurement of the rate of oxygen diffusion which indicates principally either loss of surface area of exposed blood, or some barrier to gas diffusion between alveoli and the red cell haemoglobin.

6. Direct measurement of pressure in the pulmonary artery.

7. Measurement of tensions of oxygen and $\mathrm{CO}_{2}$ in the blood.

8. Ability to measure certain aspects of lung function in each lung individuallybronchospirometry.

Recent work has emphasised the value of making some of these measurements while the patient is exercising, as this will often show more clearly the degree of defect present. This list presents a formidable array, and it is interesting to note that techniques for the measurement of Nos. 3, 4, 5, 6, 7 and 8 have all been developed within the last I 5 years, and those used to measure the remaining two have been simplified and clarified during the past few years.

\section{In What Clinical Situations is the Measure- ment of Pulmonary Function Useful ?}

It would be surprising indeed if at this stage it were possible to define clearly all the situations in which these tests of function are of value. The use of these tools in research is evident, and need not be stressed here; but much of the work necessary before a final verdict can be given on their clinical application, remains to be done. Comroe has described a few clinical cases illustrative of some of the clinical problems in which function tests may be useful, and enough has perhaps been learned to permit certain conclusions about the value of these tests in clinical medicine. The field of study being potentially so large, each worker in it has inevitably mainly given his attention to particular problems, and for this reason I will stress those situations in which personal experience has indicated the usefulness of these methods of study.

(a) In Accurate Diagnosis of Emphysema.

The pathological condition that leads to the most gross defects of all aspects of pulmonary function is emphysema. It is sometimes erroneously thought that because the classical fully developed condition is easy to recognize, therefore there is no diagnostic problem. In my experience, difficulty is likely to arise in three distinct situations.

I. If there has been minimal antecedent bronchitis. In this country, the majority of patients develop emphysema secondary to obvious chronic bronchitis, and it is therefore sometimes forgotten that emphysema may occur without any convincing history of bronchitis and without any sputum. The patients are often younger than those whose emphysema has followed chronic bronchitis, and the diagnosis may easily be missed. In two such cases in my experience, the patients have been referred to the psychiatrist, the dyspnoea having been diagnosed as 'functional.' In one other, the dyspnoea was vaguely attributed to 'left ventricular strain' (in the absence of hypertension and with a normal ECG). This type of patient does not have large bullae in the lungs, and the posteroanterior X-ray film may look relatively normal. Occasionally, these cases are seen as patients with evidence of right ventricular failure of which the cause is clinically obscure.

2. When polycythemia is present, with minimal bronchitis. There is no defect of respiratory function in patients with primary polycythemia rubra vera. The presence of bronchitis raises the question of the possibility of whether the polycythemia is secondary to emphysema. In certain patients this problem can only be finally solved by the use of function tests.

3. In the assessment of severity and response to treatment. There is evidence that the prognosis in emphysema is related to the level of impairment of gas diffusion. This defect is due to a progressive loss of surface of lung parenchyma and from the functional point of view constitutes the most characteristic impairment of emphysema. The stage of chronic bronchitis and bronchospasm is distinguished from it by the maintenance of relatively normal gas diffusion together with the same evidence of ventilatory obstruction. By the use of tests Nos. I, 2, 4 and 5 therefore, in any given patient an estimate can be made of his status in respect of irreversible change. This distinction is at the moment mainly of research value, and is of use in classifying more accurately the response to treatment of patients generally labelled as "chronic bronchitis and emphysema.' 
4. In alleged 'emphysema.' Of these, perhaps the most interesting is the study of patients who present with one large ' emphysematous' bulla in the lung. The nature of these lesions is obscure, but pulmonary function tests before and after surgery enable an accurate opinion to be given of the normality of the rest of the lungs. In my experience, sometimes the remainder of the lungs is quite normal, but in one patient, in spite of normal X-rays, there was clearly a generalized defect involving the apparently normal lungs after operation (see Fig. 3).

\section{(b) In the Study of Pulmonary Infiltrations and Fibrosis}

In this highly confused field of medicine, it is important to recognize that severe function defect may be present with little radiological change, and that evident radiological change may not be accompanied by more than slight functional defect. For instance, in following the treatment of a case of acute miliary tuberculosis, it was found that nine months after treatment was begun, the lungs were still twice as 'stiff' as normal and the rate of gas diffusion was half normal. The patient came with the comment that she had no disability and the X-rays of her lung fields was completely normal: both these comments were correct and yet the lungs had clearly not returned to normal (see Fig. 2a). The systematic study of the use of ACTH (for instance) in sarcoidosis, in chronic diffuse interstitial fibrosis, or in collagen diseases, necessarily involves the use of tests of function if it is to be complete, just as in the 'nephrotic syndrome' the effect of treatment may be followed by routine tests of renal function or plasma protein estimation. It is difficult to convince chest physicians that the following of treatment with function tests in cases of lung disease is no less and no more unreasonable than to follow the progress of renal or hepatic disease with appropriate objective tests. Certain types of pulmonary fibrosis, such as industrial pneumoconiosis, present special problems which have yet to be tackled with modern techniques of study.

(c) In the Study of the Effect of Lung Surgery and in Pre-operative Assessment

The effect of surgical procedures on lung function can only be accurately assessed by means of tests of function. A recent investigation has shown how complex is the problem of disability after pneumonectomy, and it is already clear that further work is required to clarify the relationship between 'over-inflation ' and 'emphysema.' Some of the controversial points about preferential operations from the point of view of function could be settled by a careful study of different groups of patients with the function tests now available. More complex problems, such as the results of lung denervation or the effect of removal of bullae in the lung, can quite clearly only be 气 approached intelligibly with the assistance of $z$ function tests. Bronchospirometry is fairly $\stackrel{\circ}{\complement}$ generally used on the continent in the pre-operative $C$. assessment of function of the two lungs separately. $\vec{F}$ It has not yet been used in conjunction with measurements of lung mechanics or gas diffusion, but it may be expected that these will add to its value.

(d) In the Study of Rare and Obscure Diseases It is often said that it is only in rare and $\%$ ' interesting' diseases that the use of pulmonary $\vec{\circ}$ function tests is of value. Unless the disorders mentioned above are all in this category, this is clearly not true. It is true that they are of value in obscure cases, such as arterio-venous fistula in the lung, but in my experience these cases constitute a small fraction of those in which these tests are useful.

\section{(e) As Research Investigations}

Physicians, particularly if they have no interest in nor aptitude for experimental work, are very apt to forget that all clinical investigations started life as esoteric research methods. Once the ' neighbours ' use them, as it were, they take their place as respectable clinical tools. One should not have to stress the research aspect of the use of tests of function in the light of the experimentat work published in the last ten years. There no doubt but that they will be increasingly used in the study of respiratory disorders.

\section{What Facilities and Apparatus are Required ?}

It is evident that the facilities and equipment required depends entirely on the scope of the work to be undertaken. In the case of a large hospital dealing exclusively with respiratory disease of all types, the size of the department of applied physiology should be limited only by the financial support such a department can receive-there will always be plenty for it to do. It is not, in my view, unreasonable to maintain that such major centres should be equipped to undertake complete function studies when required. The correct balance between purely research studies and purely routine investigations is not an easy one to define, but it becomes easier as physicians and surgeons understand the type of problem in which function tests are of real assistance. In my experience a $N$ minimum team for such work, able to study fully N perhaps 12 patients a week, requires one full time and one half time research worker, both fully trained, and two fully trained technicians as a minimum. It takes perhaps about a year to train someone with an aptitude for this type of work in the 'routine' studies and a further year before he is fully conversant with the field. It must be 


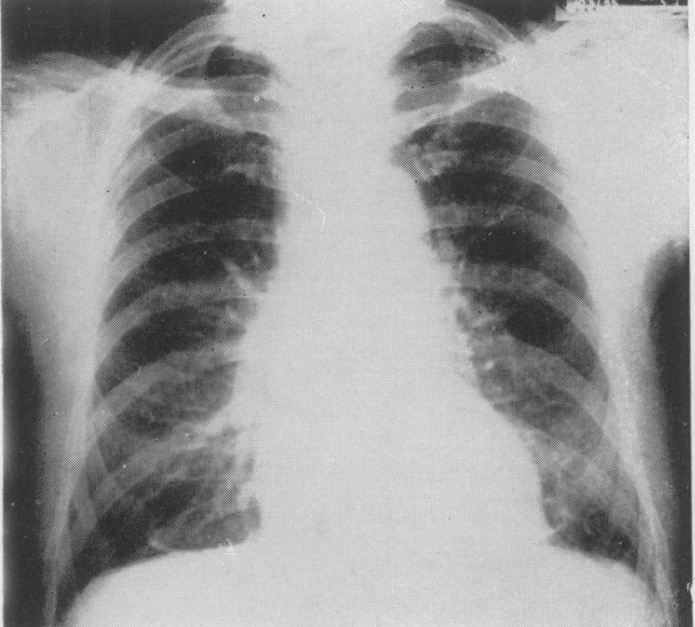

Fig. Ia

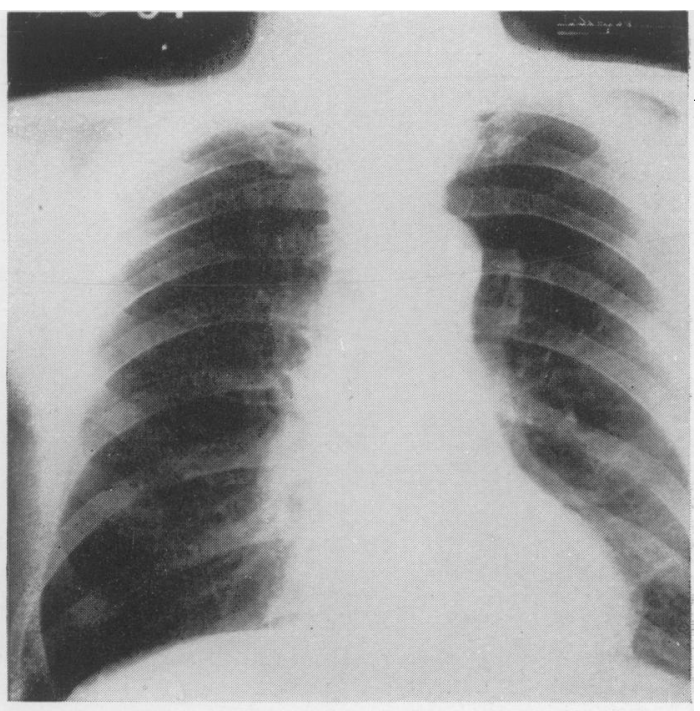

Fig. Ib

Fig. 1.-Both these $\mathrm{X}$-rays are of men in their fifties, each with polycythemia $(\mathrm{Hb}=130$ per cent.). In the case of (a), all pulmonary function tests were normal, as is usual in polycythemia rubra vera. Although the respiratory history in the case of (b) was insignificant, there was a marked impairment of gas diffusion and a reduced maximal breathing capacity-suggesting that the lung condition (? emphysema) was probably responsible for the polycythemia.

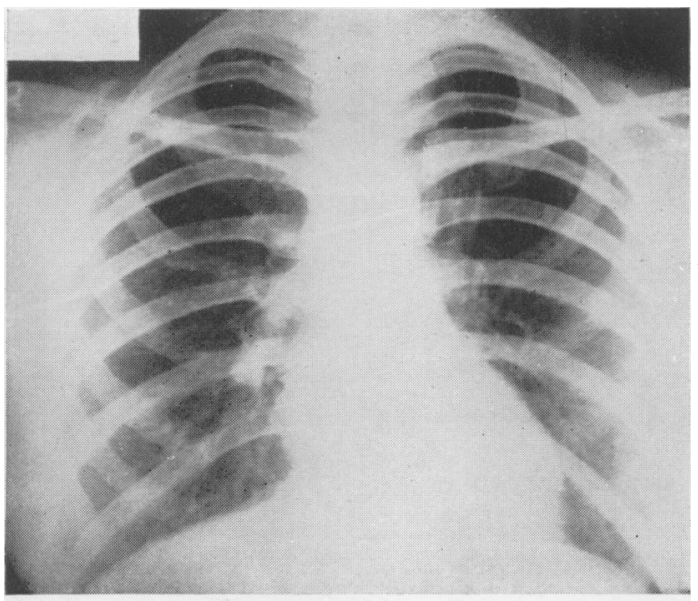

FIG. $2 \mathrm{a}$

Fig. $2 \mathrm{a}$ is the $\mathrm{X}$-ray of a girl, aged $\mathrm{I} 9$, seven months after treatment of severe acute miliary tuberculosis of the lungs. At this time she was symptom-free and this X-ray was considered to be clear. Lung function tests showed that the lungs were still twice as 'stiff' as normal and the diffusing capacity was only half the normal value.

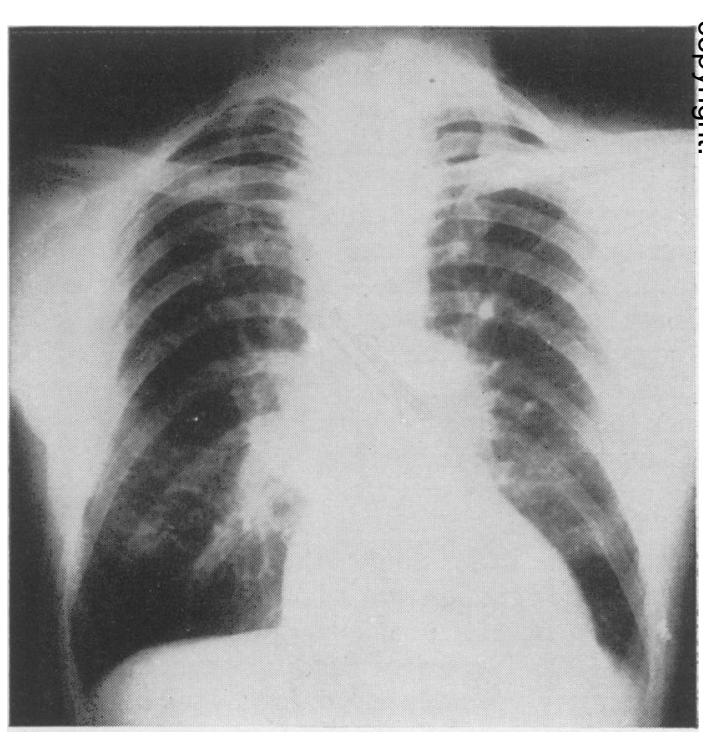

FIG. 2b

Fig. 2b.-Man, aged 57. 20-year intermittent exposure to silica (grinding). Recovered for attack of right ventricular failure. X-ray evidence of fibrosis slight but definite. Pulmonary function tests showed extreme reduction in lung compliance ( $=$ very stiff), and severe interference with gas diffusion, indicating a much more severe degree of lung change than would have been judged from the $\mathrm{X}$-ray appearance. 


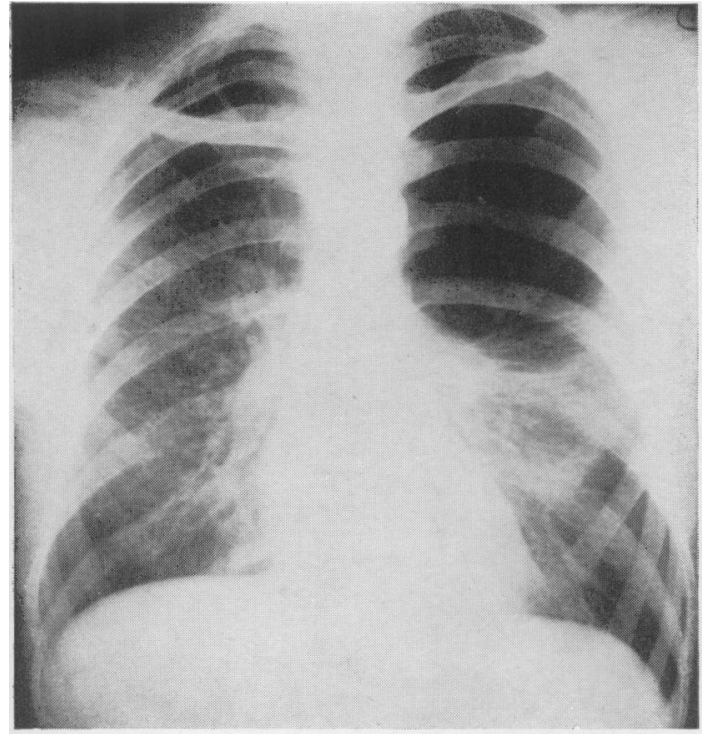

FIG. $3 \mathrm{a}$

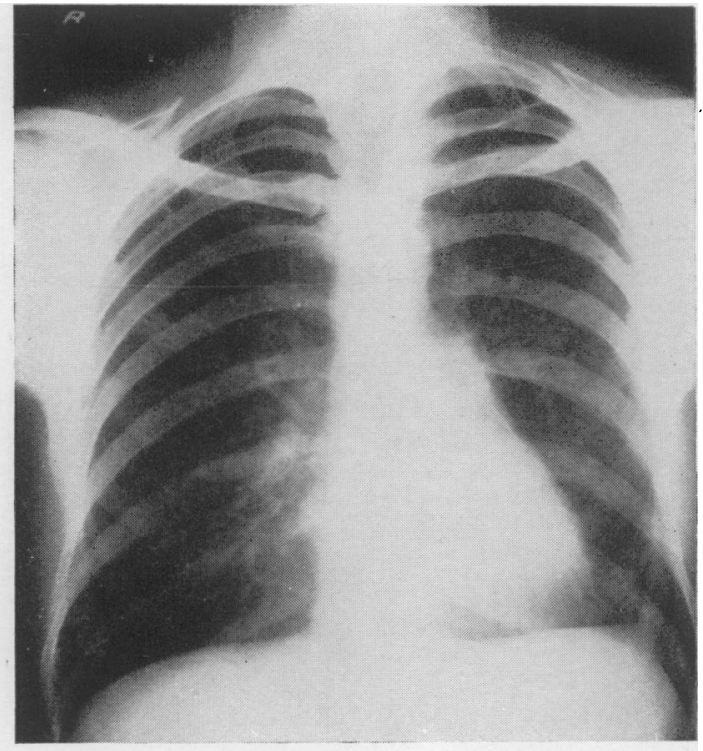

Fig. $3 \mathrm{~b}$

Fig. 3.-These X-rays are of the same man (aged 30), before and after removal of the cyst in the left upper lobe. There was a moderate degree of impairment of lung function before operation, and afterwards there was no improvement in the test results. The low rate of gas diffusion, in particular, suggests strongly that the cyst was part of a generalized lung change (? rarefaction) present in both lungs. In other similar cases, the finding of normal function post-operatively presumably indicates that the cyst is a solitary phenomenon.

emphasized that the measurement of purely ventilatory tests of function (see No. I above) by one technician, is unlikely to lead to the collection of much information of real value or assistance to the physician; and it does not seem to me helpful to suggest that the consultant should be encouraged to make these observations-however simple the equipment-in his consulting room. These single ventilatory tests are of value as research tools in the study, for instance, of the effect of broncho-dilator drugs, but in my experience their usefulness is very limited in the solution of any problem of any complexity.

\section{Conclusion}

There is little doubt that modern tests of respiratory function are of considerable value in the study, management and diagnosis (perhaps in that order) of cases of lung disease. The next 20 years will probably see an increasing application of these methods of investigation, and enable the exact scope of their use to be more precisely defined. Figs. I to 3 , show X-rays of five patients in which tests of function contributed materially to the clinical management. It may be well to end with? another quotation from Trotter: 'Moreover, with all its opportunities, observation has at its best been able to disclose but little of the fundamental processes of disease, of the exact meaning of morbid phenomena, of the precise mechanisms lying behind symptoms; whereas experiment has a remarkable series of successes of these very kinds already to its credit. In view of such considerations as these, it may well be asked whether the time has not come when it should be recognized, with all the practical consequences that would follow, that observation can no longer be regarded as able to effect serious advances in medicine.'

COMROE, J. H. and others (1955), 'The Lung,' The Year Book Publishers.

TROTTER, W., 'The Collected Papers of Wilfrid Trotter, F.R.S.,' (194I), Oxford Medical Publications, 112, 194.

BACK NUMBERS OF THE POSTGRADUATE MEDICAL JOURNAL

If any subscribers have copies, in good condition, of MARCH and APRII I952, the Fellowship of Postgraduate Medicine, 6o Portland Place, London, W.I, will be glad to purchase them. 